\title{
THE ECOLOGICAL PERFORMANCE OF JIT LOGISTICS
}

\author{
Joachim Reese, Universität Lüneburg
}

JIT logistics is widely accepted through most large national and international industries. As distances and volumes of JIT transportation grow more and more on the one hand and the vehicle load decreases on the other hand, the ecological defects of JIT are broadly discussed in the public. Nevertheless, it is shown in this contribution that the primary concern of this debate is a lack of optimization.

On the first stage a strategic implementation of a JIT concept is necessary. It is first of all an organizational problem, whether to utilize a regional forwarding agent, a JIT inventory, a good traffic center, or a pick-a-back system. Consequently, there result layout problems. loading problems, and travelling salesman problems. On the second stage it should belong to the firms' main interests to minimize ecological effects by loading and routing the vehicles to their best.

The different problem structures are modelled and discussed. Numerical examples are presented which stress that economy and ecology are not only contradictory with respect to the JIT principle. The dominance of a JIT concept depends on the way, social costs are considered. Therefore, a tax solution must be separated from solutions, when fixed costs arise. Postoptimal analysis is applied to demonstrate the stability of a JIT concept with regard to federal ecological policy. 